\title{
HOW CAN QUALITY REQUIREMENTS FOR MEDICAL SOFTWARE BE SYSTEMATICALLY FULFILLED BETTER?
}

\author{
Karin Messer-Misak ${ }^{1}$, Jeroen de Bruin ${ }^{2}$ and Sten Hanke ${ }^{3}$ \\ eHealth Institute, FH Joanneum, University of Applied Science, Graz, Austria \\ ${ }^{1}+4331654536521$ \\ ${ }^{2}+4331654536515$ \\ ${ }^{3}+4331654536526$
}

\begin{abstract}
Due to a variety of factors, among others medical and legislative requirements, the quality requirements on medical software become increasingly demanding. Several quality standards and guidelines are already in place, e.g., ISO/IEC s9126, 14598 and 25000 (SQaRE). However, there is a debate on whether these standards are not specific enough for medical software. Furthermore, their effective application for requirement gathering, analysis and monitoring can be difficult. In this paper, we propose a workflow for the application of the ISO/IEC 25010:2011 standard for the systematic collection, analysis and evaluation of requirements for various stakeholders in order to systematically better fulfill quality requirements on medical software.
\end{abstract}

\section{KEYWORDS}

Software Quality Standards, Requirements Engineering, Medical Software Engineering

\section{INTRODUCTION}

Current developments in healthcare Information and Communication Technology (ICT) include the design and development of increasingly complex integrated systems comprising both software and hardware. Technological advances in healthcare have encouraged the development of new technologies that drive connectivity across the healthcare sector. As a result, systems are more difficult to certify, more difficult to abstract from other software and hardware components, and are more difficult to understand with respect to descision making. With increasing healthcare demands, these systems will inevitably outgrow medical professionals' capabilities to deliver safe, quality care in a timely manner.

Technological innovations like mobile health solutions are met with great interest by healthcare professionals, but at present evidence on their medical efficacy, as well as quality criteria frameworks for their development and use in medical settings is still lackíng. An underlying factor that complicates medical application of such technologies resides in the legislative landscape, which is currently undergoing a process of continuous definition and re-definition of guidelines for the development and application of medical devices integrating complex software and hardware components.

A central focus that connects the legislative and medical perspectives on medical software is the requirement of delivering high quality systems, software and services. To fulfill this prerequisite, a framework is needed that comprises the following: i) quality criteria for medical software and hardware development, ii) a process to validate products against these criteria and iii) a process to monitor the development process and to track the quality criteria during the project.

In the end, the assessment of the effect of software quality on patient treatment quality, healthcare institution efficiency and certain applications like clinical trials is essential. The aim of the paper is to introduce a method how to apply well-established methods from quality management as a process, which might also be used in the medical domain to control, manage and evaluate systems against certain requirements. 


\section{CURRENT GUIDELINES AND STANDARDS}

Although standards that define quality criteria for software and hardware have already been constructed, their applicability to medical software and systems is still a matter of debate; there is a discussion ongoing about the criteria in general, because application and software quality is a multifaceted concept determined by a number of properties (Aghazadeh, S. et al., 2015).

Over time, different qualitative models have been proposed to describe and delineate the different system and software quality attributes. The ISO/IEC standards 9126, 14598 and 25000 (SQaRE) are widely referred to as standard frameworks for developing complementary or alternative models for evaluating specific software or developing custom models.

The ISO/IEC 9126 standard has been introduced in 1991 by International Standard Organization (ISO) and International Electro-technical Commission (IEC). This model is used for the definition and integration of various characteristics of software quality, and has been used to asses impact of software quality characteristics on healthcare outcome (Ordozgoiti, A. V. et al., 2016). More recently, ISO/IEC 25000 standard is considered more suitable for evaluating the quality of ICTs for healthcare use from the perspective of institutional acquisition (Ordozgoiti, A. V. et al., 2016). ISO/IEC 25000 attempts to harmonize, unify and update the international standards for evaluating software quality.

Additionally, there are some non-ISO models that mostly standardize reference works and most of them are impractical and inappropriate for healthcare institutions, since they base their models on life cycle of software under development.

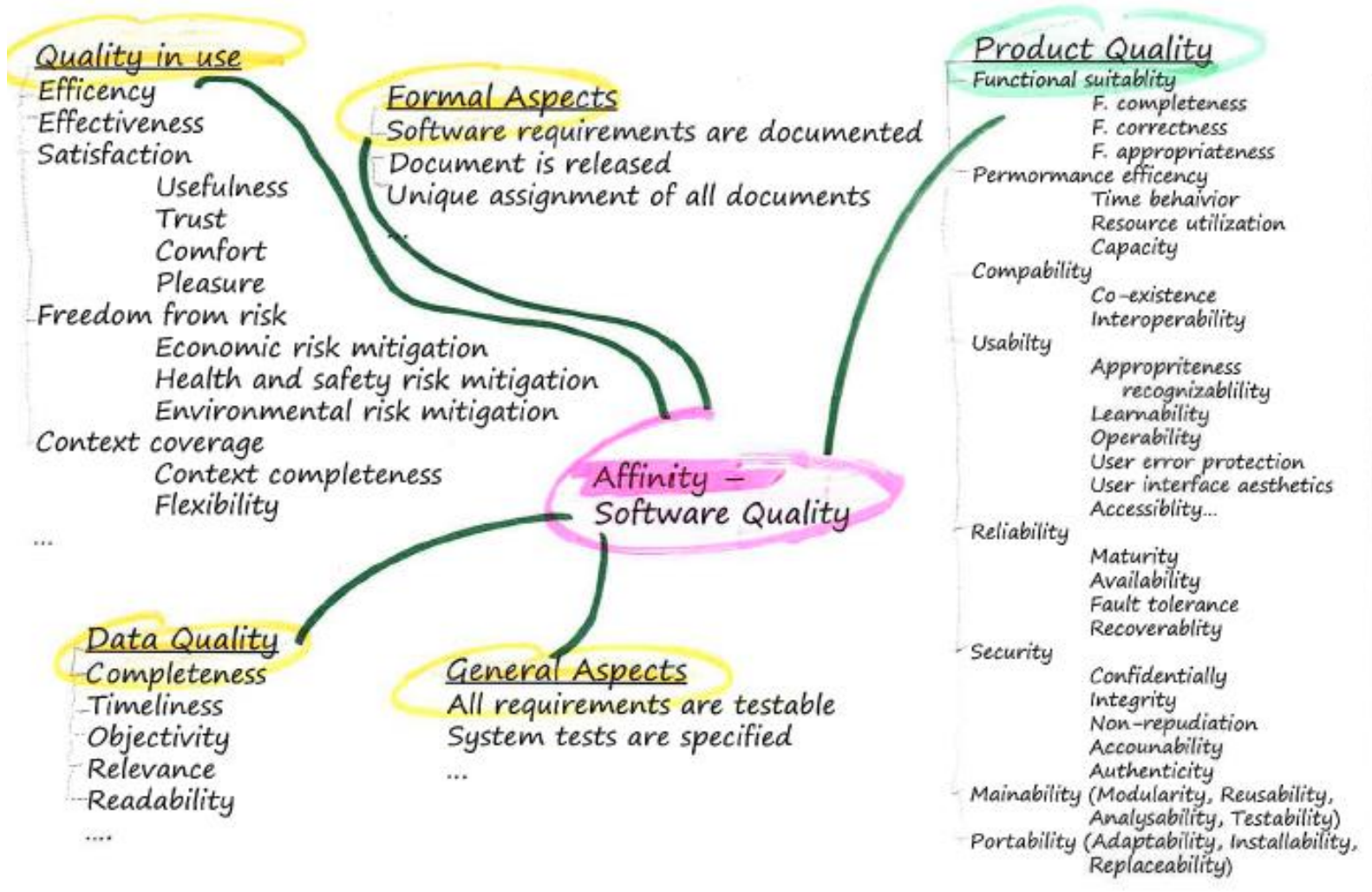

Figure 1. Affinity Diagram

Software quality requirements can be complex and their application and priority depend on the application under consideration, as well as the target application environment and stakeholders. These criteria are normally assorted in functional and non-functional requirements. For example Aghazadeh et al. (2015) indicated sixhealthcare indicators in relation to software quality characteristics based on a questionnaire with health experts. The characteristics are 1) User satisfaction, 2) Quality of patient care, 3) Clinical Workflow and Efficiency, 4) Care providers communication and information exchange, 5) Patient satisfaction and 
6) Care costs (Aghazadeh, S. et.al., 2015). Nonetheless, although requirements can vary depending on the application and application field, a process is needed to evaluate the system against these requirements.

Identifying software quality requirements early in the software life cycle as a part of the requirements specification is considered crucial. At present, quality requirements are commonly elicited from literature and experts. The standard ISO/IEC 25030 (as part of the ISO/IEC 25000), which is part of the SQuaRE series of International Standards, aims to improve the value of software quality requirements by providing guidelines and recommendations for quality requirements, and guidance for the processes used to define and analyze these requirements (Idri, A., Bachiri M., and Fernández-Alemán J.L., 2016). In particular, ISO/IEC 25030 provides a guide with which to identify software quality requirements, to validate the completeness of requirements specification and to identify quality assurance and acceptance criteria for a software product. Examples of the application of this guide include Alves et al. (2016, pp. 30-35) who validated a poison central system as well as Kadi et al. (2016) who evaluated the quality requirements of a pregnancy monitoring system, both using the ISO/IEC 25010 standard.

\section{USE OF QM METHODS IN PRACTICE}

The following activities can benefit from the use of tools known from quality management (BSI Standards Publication, 2011, p.1):

- Identification of software and system requirements;

- Validation of the completeness of a requirement definition;

- Identification of software and system design objectives;

- Identification of software and system test objectives;

- Definition of quality characteristics and control criteria in the context of quality assurance;

- Identification of acceptance criteria for a software product and/or software-intensive computer system;

These activities, methods and tools must be carefully coordinated and comnistently used throughout the entire product development process in order to ensure that the required quality requirements can be verified in the final product.

In order to identify the optimal set of software and system requirements and verify them for completeness, a comprehensive analysis of stakeholder requirements must be carried out. These requirements are to be specified as criteria, i.e. described in concrete terms, and measurement methodologies or metrics need to be defined for evaluation purposes. In order to achieve accurate and comprehensive results, it is recommended to work on this process in a stepwise fashion in interdisciplinary teams, and to employ quality management tools and methods .

\section{STEP-BY-STEP IMPLEMENTATION USING A PRACTICAL EXAMPLE}

In this section, the process will be presented step by step and proven tools that support the process will be described.

First, requirements need to be collected from all stakeholders, as they are likely to vary between stakeholders. We can distinguish between primary users (person who interacts directly with the system), secondary users (i.E. support or content provider, system manager, security manager, analyzer, installer) and sometimes indirect users (person who receives output without interacting with the system) (BSI Standards Publication, 2011, pp. 5-6).

Usually s survey of the requirements of the various stakeholders is employed, for example, with the help of an affinity diagram. Complete sets of requirements are most likely to be achieved with a collaborative working method that is carried out step-by-step. This enables user and system requirements, design requirements, etc. to be comprehensively identified, named, and categorized. The relationships between the individual information groups become transparent. In software development, it makes sense to use the models 
"Quality in Use" and "Product Quality" and their attributes mentioned in ISO/IEC 25010:2011 and then to extend and supplement them accordingly (see Figure 1).

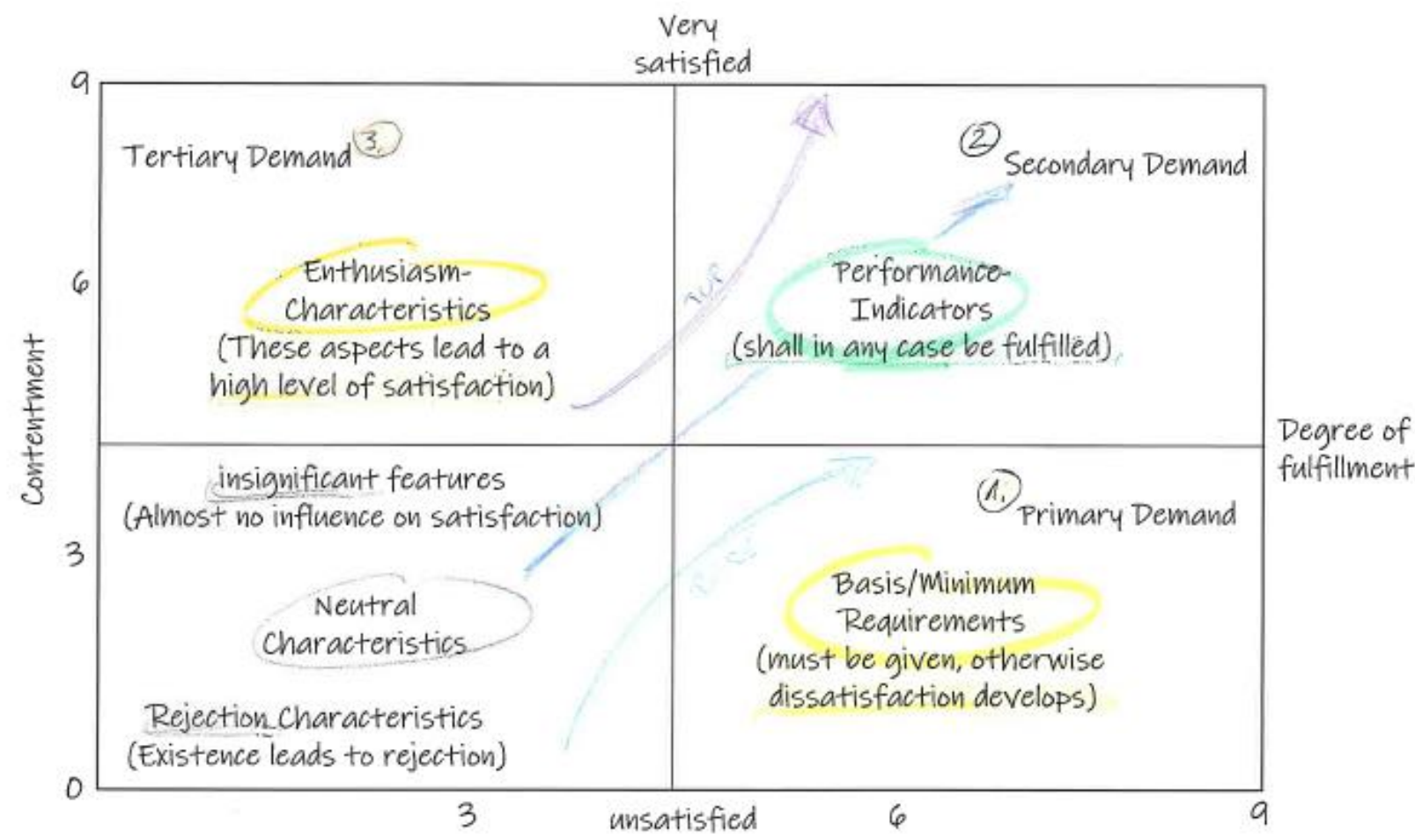

Figure 2. Kano Analysis

After requirements are collected, the information gained from this is supplemented by an affinity diagram, in which the requirements are further specified from the stakeholder/user perspective using a Kano analysis. In this process, the relationship between the achievement of certain characteristics of the requirements and the satisfaction expected by the stakeholder/user is established.

After this information supplementation phase, the requirements can be divided into 5 categories. As implicit must criteria, which are not articulated concretely by the stakeholders, but are tacitly presupposed by this, are so-called basic characteristics. Performance characteristics are requirements explicitly demanded by the stakeholders, which have a high influence on satisfaction. Enthusiasm characteristics donate felt or actual auxiliary use, which is not expected by the stakeholders explicitly, but whose satisfaction increases to a large extent. Further one distinguishes between insignificant characteristics (thus characteristics, whose existence leads neither to satisfaction nor to the dissatisfaction) and rejection characteristics (these are characteristics, which, if they exist with the user/client dissatisfaction cause, however with not being present also no satisfaction create). Each requirement is assigned to a category before it is further specified (see Figure 2).

Next, stakeholders requirements are translated into specifiable and measurable quality characteristics. In this process, the target criteria to be achieved are defined, control criteria for quality assurance are defined and the acceptance of the software product is presented in a matrix.

The tool "Quality function deployment" or "House of Quality" is suitable for planning. Especially when interfaces and contradictions occur, these can be easily identified by correlations. It shows "what" the stakeholders want and "how" the requirements are technically implemented. The method is supplemented by target values to be defined, which are defined by fixed values in a corresponding unit.

Here, too, a systematic step-by-step approach is recommended (see Figure 3):

- After the structuring and weighting of the requirement analysis results, these are displayed on row level. By weighting these entries (e.g. between 1-9) from the Kano analysis, the relevance from the point of view of the stakeholders is represented. 
- The column level lists how these requirements are met. In the next step, the links between rows and columns are displayed. For simplicity's sake, the rating is bucketed in 4 relationship levels (no relationship, weak relationship, medium relationship or strong relationship) between the requirements and the functions or technical specifications. The values are determined using the correlation matrix (weighting * relevance) both horizontally and vertically. As a result, the evaluation of the stakeholder requirements with regard to the functions on the horizontal side and the evaluation of the functions on the vertical side can be seen at a glance.

- In order to check the functions for mutual influences and dependencies, both the stakeholder requirements and the functions can be correlated with themselves.

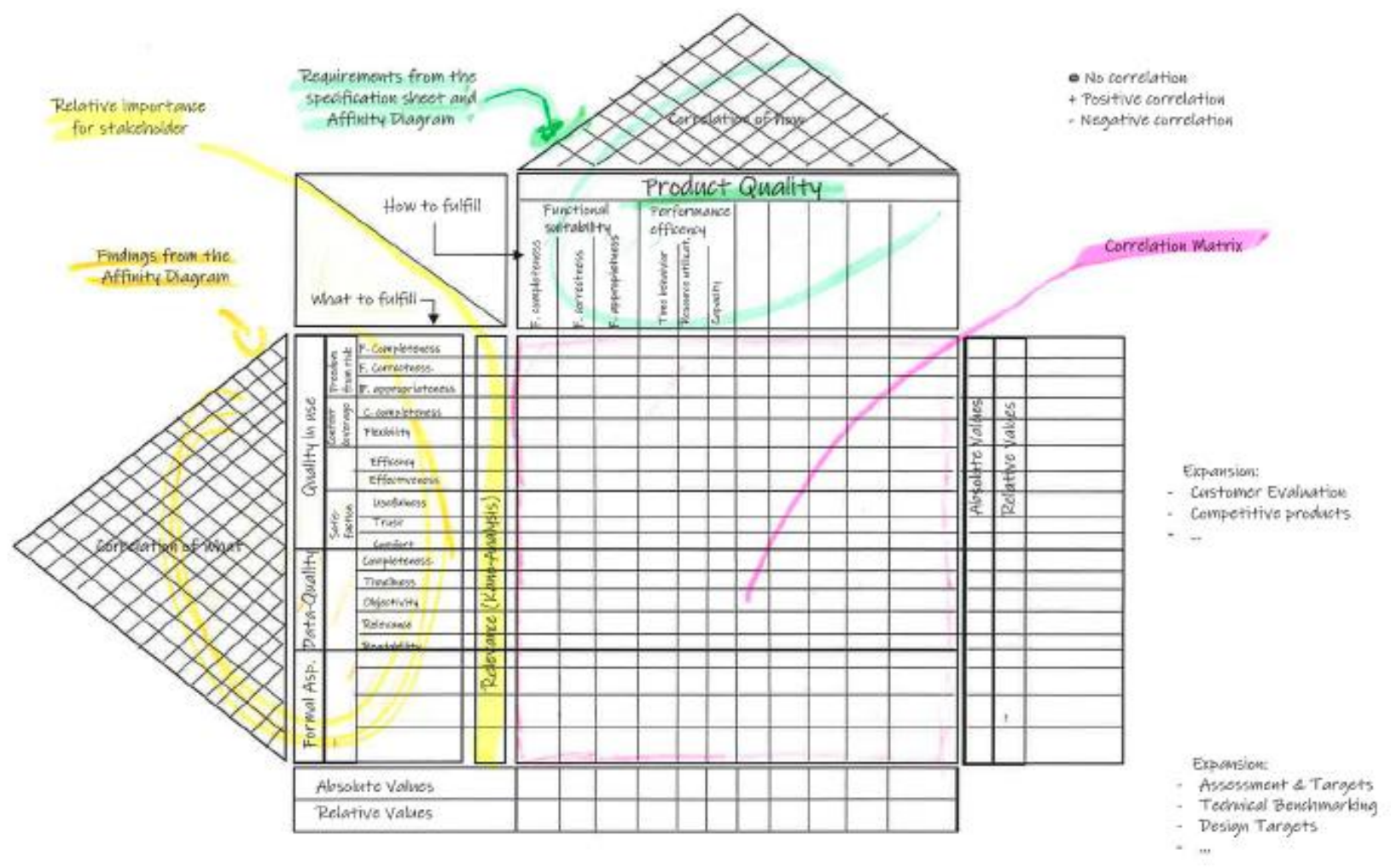

Figure 3. Quality Function Deployment

The method can be extended as required and can be flexibly adapted to suit specific requirements. Yoji Akao - one of the developers of the method - coined the sentence "Copy the spirit, not the form" (Möhrle, M.G. et al., 2007, p. 146). Herzwurm, et al. (2003) show that it makes sense to adapt existing methods and tools accordingly and to adapt them to the requirements.

\section{SUMMARY}

The demand that high-quality software systems to be realized within the planned time and at the estimated costs led to the adaptation of the methods in software development (Hohler, B., 2014, p. 831) The quality aspect is an essential factor in ensuring security and stakeholder satisfaction, i.e. success in the use of software products, especially in health care. Potential negative effects should be excluded from the outset and positive aspects should be identified and promoted. Quality characteristics must be known, specified, measured and evaluated. 


\section{REFERENCES}

Aghazadeh, S., Pirnejad, H., Aliev, A. and Moradkhani A. (2015). "Evaluating the effect of software quality characteristics on health care quality indicators," Indicators, vol. 2, no. 3, pp. 67-73.

Alves J.M. et al. (2015). "Quality evaluation of poison control information systems: A case study of the DATATOX system," in Proceedings - IEEE Symposium on Computer-Based Medical Systems, vol. 2016-August, pp. 30-35.

BSI Standards Publication (2011). Systems and Software engineering - Systems and Software Quality Requirements and Evaluation (SQuaRE) - System and software quality models, BSI, 31.3.2011, p. 1.

BSI Standards Publication (2011). Systems and Software engineering - Systems and Software Quality Requirements and Evaluation (SQuaRE) - System and software quality models, BSI, 31.3.2011, pp. 5-6.

Herzwurm, Schockert, Dowie u. Breidung (2003). Requirements Engineering for Application Development in Volatile Project Environments Using Continuous Quality Function Deployment (CQFD), in Proceedings of the International Conference on Software Engineering Research and Practise, Vol. 1, pp. 440-447.

Hohler, Bernd (2014). Qualitätsmanagement bei der Softwareentwicklung, pp. 827-866 in: Masing Handbuch Qualitätsmanagement, Carl Hanser Verlag, München, Wien, p. 831.

Idri, A., Bachiri, M. and Fernández-Alemán, J.L. (2016). "A Framework for Evaluating the Software Product Quality of Pregnancy Monitoring Mobile Personal Health Records,” J. Med. Syst., vol. 40, no. 3, pp. 1-17.

Kadi, I., Idri, A. and Ouhbi, S. (2016). "Quality evaluation of cardiac decision support systems using ISO 25010 standard," in Proceedings of IEEE/ACS International Conference on Computer Systems and Applications, AICCSA, 2016, vol. 0.

Möhrle, Martin. G., Hartung, Knud and Spilgies Wulf-Dieter (2007). Quality Function Deployment for bundled services., in Innovation Management in the Service Industry. Basics, practical examples and perspectives. Edited by Klaus Schmidt/Roland Gleich/Ansgar Richter. pp.139-160, Haufe Publishing House, Freiburg. 2007, p. 146 - quotes Akao 1992, p. 19.

Ordozgoiti, A.V., Hito, P.D., Guix Comellas, E.M., Sanchez, C.M.F., Hernandez M.G., and Canut, T.L. (2016). "Software quality evaluation models applicable in health information and communications technologies. A review of the literature," in Studies in Health Technology and Informatics, vol. 226, pp. 169-172. 